\title{
Why is Principlism limited in its' Utility in Psychiatric Settings?
}

\author{
Srikanth Nimmagadda ${ }^{1}$, Charlotte Caton ${ }^{2}$, Kikelomo Anunobi ${ }^{3}$, Kathryn Crossley ${ }^{4}$, \\ Jonathan Glynn ${ }^{5}$, Claire Buchan ${ }^{6}$ \\ ${ }^{1}$ Consultant Forensic Psychiatrist, Cheswold Park Hospital \\ ${ }^{2}$ Forensic Psychologist, Cheswold Park Hospital ${ }^{3}$ \\ Speciality Doctor, Cheswold Park Hospital \\ ${ }^{4}$ Social Worker, Cheswold Park Hospital \\ ${ }^{5}$ Occupational Therapist, Cheswold Park Hospital \\ ${ }^{6}$ Ward Manager, Cheswold Park Hospital
}

\begin{abstract}
Principlism is one of the most influential approaches to bioethics in the modern times. The application of the four principles to ethical dilemmas in psychiatric settings permits some analysis but not necessarily, a complete resolution of the problems. The authors acknowledge that there is discussion by the principlists of what should be considered when the principles conflict, however, there is no clear cut approach in addressing various dilemmas in a systematic way in the event of the clash of the principles. So, while the principlism approach might enable us to describe a moral dilemma as a conflict between competing principles, it will not necessarily dictate any particular outcome. The authors highlight this point by discussing a specific ethical dilemma encountered in their clinical practice. Beauchamp and Childress's approach of resolving the conflict between two principles by using the model of specification is far from satisfactory. It does not specify which conditions in the specification model to be considered as very important in arriving at a solution. Also, if all conditions are given equal importance, what happens if only two or three conditions are met adequately to justify infringement of the principles. Authors conclude that principlists take a narrow approach and fail to consider a host of other arguments and issues when arriving at a solution to various ethical and moral dilemmas in clinical settings.
\end{abstract}

Key Words: principlism, ethical dilemma, moral theory, autonomy, non-maleficence, beneficence, capacity, consent

\section{INTRODUCTION}

Beauchamp and Childress advocated a four principle approach known as 'Principlism', which offered to guide in medical decision making [1]. In this article, the authors would like to argue their case that principlism does not offer much help in the event of conflict of the principles. They would like to illustrate this by highlighting a clinical scenario that they encountered in their clinical practice in a medium secure ward at Cheswold Park Hospital (a secure psychiatric hospital based in Doncaster, United Kingdom).

The four principles include:

1. Respect for Autonomy: According to this principle, patients should be treated as rational, autonomous agents, making their own decisions about their lives. 
2. Non-maleficence: The principle that health care professionals should do no harm to their patients.

3. Beneficence: The principle that the health care professionals should only do "benefit" for their patients and to balance benefits against risks.

4. Justice: This principle is not retributive but distributive, and concerned with how scarce healthcare resources should be shared fairly between individuals.

\section{ANALYSIS AND CRITIQUE OF PRINCIPLISM}

The four principles' approach had its origins from some important judgments and approaches in the common morality and medical traditions. Beauchamp and Childress argued that all the prima facie principles are equal and universal and do not consider that any principle has any priority over all other principles. Gillon argued that the approach is "compatible with a wide variety of moral theories" and because it uses shared primae facie common moral norms, it helps us to avoid two polar dangers, moral relativism and moral imperialism [2].

Principlism uses features of various ethical theories that have a good support in the field of bio ethics. For example, in relation to the principle of beneficence, it acknowledges that Mill was right in being concerned with the consequences. Similarly, whilst addressing the principle of autonomy, it argues that that Kant was right in attaching importance of the individual person. However, it does not make any attempt to combine various concerns arising from these into a single adequate theory, rather than disparate concerns derived from several competing theories [3]. Also, principlism is criticized because the four principles can be accepted and used by other moral theorists (like deontologists and consequentialists), they do not provide an explanation of what morality is and a guide for answering moral questions and resolving moral dilemmas. Therefore, it can be argued that the four principles might act as intermediary levels of ethical reasoning between individual doctor- patient encounter and abstract moral theory.

It is argued that despite tendencies to compete for a prime place in moral theory, the four principles approach should not claim to be superior to other moral theories [4].

The advantages of principlism are that it is simple and universal in application and the disadvantages include neglect of emotional and personal factors, oversimplification of the issues, and excessive claims touniversality.

Professor Gillon has advocated the use of the Beauchamp and Childress for many years and emphasised that the four principles approach is a widely and interculturally acceptable method for medical ethics analysis [5]. Danner Clouse and Gert argued that rather than clarifying difficult questions, principlism may be unsystematic and misleading. They further stated that "principles of biomedical ethics" approach is mistaken and misleading. Principlism is mistaken about the nature of morality and is misleading as to the foundation of ethics...."[6]

In general, it can be argued that when confronted with a moral dilemma, there would be a conflict between two or more of these basic principles from different angles. This causes considerable problems to health care professionals and leads to considerable debate and anxiety that the principles (which they fully acknowledge and accept) are in conflict with each other.

\section{APPLICATION OF PRINCIPLISM TO AN ETHICAL DILEMMA IN A CLINICAL SETTING}

We shall highlight an ethical dilemma that we encountered in our clinical practice that resulted in the conflict of various principles. The authors work in a secure psychiatric inpatient unit and we have a patient $\mathrm{Mr} \mathrm{B}$, suffering from a mental disorder, namely, Emotionally Unstable Personality disorder, Borderline type). $\mathrm{Mr} \mathrm{B}$ is detained under the provisions of the Mental Health Act 1983. Mr B displayed long-standing pattern of maladaptive behaviours pervasive across a wide range of personal and social situations and associated with personal distress. $\mathrm{Mr}$ 
B's symptoms and behavioural patterns of his personality disorder were characterised by a marked tendency to act impulsively, mood fluctuations, emotional instability, difficulty in maintaining any course of action that offers no immediate reward, an uncertainty about personal and sexual identity, liability to become involved in intense and unstable relationships. Also, significantly his presentation is characterised by recurrent threats and attempts of self-harm and suicidal behaviours by tying ligatures on numerous occasions. He also displayed aggressive and violent behaviours towards others and also damaged property on numerous occasions.

Mr B was prescribed various psychotropic medications for his symptoms, offered psychological interventions and encouraged to attend various groups and activities to improve his self esteem, confidence and also to aid in his independent living skills. Following a serious suicidal attempt, the clinical team discussed his presentation in great detail and concluded that $\mathrm{Mr} \mathrm{B}$ needed to be maintained on a robust management plan. Mr B's management plan included continuous 24 hour one to one nurse monitoring and observation both in his bedroom and ward areas. In addition, he would be supervised by nursing staff in his bathroom and toilet, his room removed of most of the belongings as a precautionary measure, so that he would not use any items (for example, he can use clothing as a ligature and any sharp item to cut himself) for harming himself. As a part of the treatment plan, it was agreed by the clinical team that in the event of any violent and aggressive behaviours, $\mathrm{Mr} \mathrm{B}$ could be administered intramuscular antipsychotic and anti anxiolytic medication without his consent. However, Mr B protested in relation to this management plans and argued that it would make him feel distressed and also, violate his privacy and dignity. He also continued to express active suicidal thoughts and maintained that that he was ambivalent about the consequences of his behaviours, even if this led to death. $\mathrm{He}$ strongly objected to this management and treatment plan.

In the above example, there is clearly a conflict between the beneficence and non-maleficence. The requirement of beneficence is to "do good" or to promote wellbeing of the patient and the clinical team to a large extent would achieve this by implementation of the strict treatment plans. The robust management plans would help in constantly monitoring Mr B's mental state and ensuring his safety. The risk of not having these plans may result in serious incident including suicide or harm to others. On the other hand, there is maleficence as a result of the imposition of various restrictions of the management plans. For example, there is invasion of the patient's privacy and dignity and this would affect the patient's self esteem considerably and would arouse feelings of shame and guilt. Also, the management plans may cause further psychological harm by evoking memories of some of the trauma issues (including abuse in his childhood) and thus, perpetuating his negative cognitions and behaviours.

Beauchamp and Childress acknowledged that when principles conflict, there are no readymade solution except to decide which principle is more important in that particular situation. Although Beauchamp and Childress discussed the concepts and obligations in relation to beneficence and non-maleficence, they do not exactly offer any solution in the event of clash between the 2 principles.

In the above moral dilemma, adopting a balanced approach, it was concluded by the clinical team the principle of beneficence would carry more weight and was a higher priority than nonmaleficence. Thus, it can be argued that in the moral dilemma involving conflict between two or more competing principles, principlism approach would adopt a balancing approach, but it failed to give a definite solution or outcome. It was suggested that a possible solution might be to rank the principles in a hierarchy, but however, this would require justification, which is not provided by the principlism approach itself [6].

In relation to the principle of beneficence, the clinical team may have acted in a paternalistic way in deciding what is best for the patient. It can be argued that the clinical team is not taking into account the patient's views, who may be possibly competent (although, the capacity may be 
fluctuating) as to what is best for himself. Although the patient may be competent, the principle of beneficence would trump all the principles because the clinicians dealing with detained patients will be acting within the constraints of the legal statutory framework emphasising significantly on issues like dangerousness and protection of others.

Although principlists offer a balancing model and further, a specification approach in relation to addressing the conflicts between various principles, it is far from satisfactory in dealing with moral dilemmas that I encountered in my psychiatric practice.

Beauchamp and Childress also acknowledged that the framework of the 4 principles approach helps to identify and reflect on moral problems. However, the framework does not contain sufficient content to address the nuances of many moral circumstances. It was argued by opponents of principlism that the balancing approach of the principles exposed the weakness of the principlism of being open-ended and lacking adherence and commitment to firm principles [1]. Beauchamp and Childress responded to their critics and introduced the concept of "specification". They advocated that "Specification is a process of reducing the indeterminateness of abstract norms and providing them with action guiding content." They further added "Specification entails a substantive refinement of the range of scope of norms, whereas balancing consists of deliberation and judgment about the relative weights or strength or norms. Balancing is especially important for reaching judgments in individual cases, and specification is especially useful for policy development ..." [1]

Beauchamp and Childress listed 6 conditions that must be met to justify infringing one prima facie principle in order to adhere to another [1]. We would like to discuss 2 of the 6 conditions set out by Beauchamp and Childress which must be met to justify infringing one principle in order to adhere to another. For example, one of the conditions, (condition 2) specified by Beauchamp and Childress is "The moral objective justifying the infringement must have a realistic prospect of achievement." It could be argued that in the moral dilemma highlighted above, it is highly debatable to achieve any prospect of progress because any solution is temporary and it depends on the characteristics of the patient, severity of his mental disorder and his willingness to engage and cooperate in various treatments. Another condition (condition 5) stated, "The agent must seek to minimise any negative effects of the infringement". Considering the above dilemma, it is difficult to predict the negative psychological impact of the infringement (i.e., beneficence over non maleficance) and it sequale in the future, as it might evoke a whole range of psychological emotions including feelings of shame, disrespect, anger and hostility towards him and society. Similarly, the other four conditions specified would lead to some difficulty in explaining the infringement of the principles.

There is no doubt that principlism provides a useful "checklist" approach to bioethics. We concur with the view of John Harris, a critic of principlism that in addressing moral and ethical dilemmas, it is just not sufficient to identify the principle relevant to the particular dilemma, but, to consider analysing the arguments that are being debated in providing the solution [7].

In the above example, the clinical team had no problem identifying the underlying principles that were in conflict, but engaged in a serious debate in arriving at a solution, which was not morally, or ethically questionable. Although a balancing approach is considered which, is the usual way of arriving at a solution in the event of conflict of the principles it means that many strands of the analysis of the argument (in relation to the moral dilemmas) would be missed or ignored in order that one principle trumps over other principle. Thus, it cannot be said that any solution arrived as a result is absolutely morally right.

Discussion will now by focused on the principle of autonomy before considering its' importance in the moral dilemma of the above case. In relation to the principle of autonomy, Beauchamp and Childress noted, "To respect an autonomous agent, is at a minimum to acknowledge that person's right to hold views, to make choices, and to take actions based on personal values and beliefs..." [8] 
Gillon, a noted principlist argued that autonomy is the "first among equals" [5]. He argued that autonomy is the key factor in ensuring that morality is possible and also, principles of "beneficence and non-maleficence to other autonomous agents both require respect for the autonomy of those agents." He further argued that justice is also a necessary component of the principle of autonomy because it takes into account the autonomous views of the people in trying to meet their needs and demands for resources. However, it was argued that Gillon's special emphasis for the principle of respect for autonomy contradicts his own view that the principles are prima facie universal moral principles [9].

Gillon also expressed as a result of the importance of autonomy, cultural variations can play an independent normative role in the development of the moral judgments. Dawson and Garrard did not accept that respect for autonomy is first among equals as a moral principle. They also argued that this special significance (as attributed by Gillon) to the principle of autonomy does not offer any independent moral role for cultural variation [10]. They rejected the plea from Gillon that there needs to be middle ground: between moral relativism and moral imperialism. Furthermore, Dawson and Gerrard emphasised that they disagreed with Gillon's claim that the respect for autonomy would outrank other principles, particularly the principle of nonmaleficence. They emphasized tolerance as an important universal moral notion and leaned towards moral objectivism.

In our view, although Gillon emphasised the importance of autonomy and argued it is "first among equals", there is no significant analysis in the approach, and thus, not applicable to moral dilemmas in all situations. Furthermore, Gillon failed to address the interplay of conflict between other principles other than autonomy. Although the principlists (like Gillon) or its critics offer various perspectives to the approach of principlism, they do not offer any further guidance or useful analysis in addressing the conflict of the principles in solving the moraldilemmas.

Again, referring to the case of Mr B highlighted above, there is also clash between principles of autonomy and beneficence. Here, the autonomy, i.e., the views and wishes of the patient were not in line with the clinical team's opinion of what was in the best interests of the patient. However, the conflict between these 2 principles is less important in Mr B's case as he is detained in the hospital for treatment under a section of the Mental Health Act 1983. Under the Mental Health Act 1983, a person can be treated for their mental disorder without any need for a regard of whether an individual is competent to refuse treatment. However, the situation might be slightly different if $\mathrm{Mr} \mathrm{B}$ was an informal patient in a psychiatric unit. In this scenario, given his presentation, is the same as described above, the principles of respect for autonomy, beneficence and non- maleficence would have competed with each other. Again, a similar balancing approach would have been adopted to ascertain which principle takes precedence over others.

In the event of clash between principles of autonomy and beneficence, it is argued that if the beneficence is understood in terms of how the patient regards what is the best treatment for him, (for example, if the patient has insight and when mentally well at some point, believes that the management approaches by the clinical team are effective) the potential conflict is lessened [11]. However, a counter argument is that if the harm or benefit is determined by the patient, then these concepts would be considered to essentially equate to the principle of autonomy.

Many ethical problems in psychiatry revolve around the issue of capacity. A patient's autonomy may assume that the patient's capacity to make rational choices is not impaired, either temporarily or permanently by mental or physical disorders. If capacity is limited or impaired, what principles then take over to guide action? Is the principle of beneficence in the form of paternalism implied in best interest standard the right approach to be adopted? These questions are not properly addressed by the principlists when they adopt a narrow approach of applying balancing approach model. 


\section{CONCLUSION}

In conclusion, principlism is a useful approach, which provides a checklist of factors and a framework in approaching various moral and ethical dilemmas. There are some advantages to the principlism approach. For example, it is easy way to address problems that arise in healthcare settings. Also, these principles are universally accepted and thus ensure consistency in approaching various moral dilemmas (by balancing all the different principles.) However, despite claims of the universality nature of the principlism approach, it does not take into account the role of other factors, for example, principle of respect for autonomy being affected by compulsory detention of patients in psychiatric settings.

Although Gillon has adopted a view and attached a special significance to the principle of autonomy over other principles, his justification cannot be applied to number of moral dilemmas in clinical decision making. Also, he does not address the issue of what happens if there is a clash between two other principles other than autonomy (for example beneficence and nonmaleficence). Although principlists have worked on the framework of addressing moral dilemmas in few situations, they have so far been unable to address the conflict of the principles in various ethical and moral dilemmas in various situations. For example, they failed to highlight a solution to a moral dilemma in a secure in-patient psychiatric setting where risk assessment and risk management form a cornerstone of clinical practice. In addition, they do not consider issue of the personal autonomy being attached less importance as a result of the deprivation of liberty due to a mental disorder and do not offer any approach to clinicians encountering such problems.

The authors conclude that the Beauchamp and Childress's approach of resolving the conflict between two principles by using the model of specification is far from satisfactory. It does not specify which conditions in the specification model to be considered as very important in arriving at a solution. Also, if all conditions are given equal importance, what happens if only two or three conditions are met adequately to justify infringement of the principles. We conclude that principlists take a narrow approach and fail to consider a host of other arguments and issues when arriving at a solution to various ethical and moral dilemmas in clinical settings.

\section{REFERENCES}

1. Beauchamp TL, Childress J. Principles in Biomedical Ethics (5th ed) Oxford, New York: Oxford University Press; 2001.

2. Gillon R. Ethics needs principles; four can encompass the rest and respect for autonomy should be first among equals. J Med Ethics 2003;29;307-12.

3. Jackson E. Medical Law: Text, cases and materials and Ethics, New York, Oxford University Press ; 2005.

4. Campbell AV. The virtues (and vices) of the four principles. J Med Ethics 2003;29:292-6.

5. Gillon R. Four scenarios. J Med Ethics 2003;29:267-8

6. Jackson EA. Critique of Principlism. J Med Philos 2006;2;16-7.

7. Jackson E. Medical Law: Text, cases and materials and Ethics, New York, Oxford University Press ; 2006.

8. Beauchamp T. Methods and Principles in Biomedical Ethics. J Med Ethics 2006;29:269-74

9. Harris J. In praise of unprincipled ethics. J Med Ethics 2003;29:303-6.

10. Dawson A, Garrad E. In defence of moral imperialism. J Med Ethics 2006;32:200-4

11. Herring J. Medical Law and Ethics, Oxford, Oxford University Press ; 2005.

Acknowledgements - Nil

Source of Funding - Nil

Conflict of Interest - Nil 